\title{
Interdisciplinarité et production de connaissances : quelles recherches voulons-nous?
}

La production des connaissances est affaire d'hommes et d'institutions. Dans les trois dernières années, plusieurs événements ont concouru en France à en transformer les conditions d'exercice : les états généraux de la recherche qui, en 2004, ont nourri de riches débats au sein d'une communauté mobilisée dans le cadre du mouvement «Sauvons la recherche » (SLR); la mise en place de l'Agence nationale de la recherche (ANR) avec, à ses côtés, l'Agence d'évaluation de la recherche et de l'enseignement supérieur (AERES); la loi d'orientation et de programme, vingt ans après la loi Chevènement ; la mise en place des pôles de recherche et d'enseignement supérieur (PRES) et des campus de recherche dans les universités, des réseaux thématiques de recherche avancée et des fondations. . .

Les débats, les controverses, les prises de position, les crises institutionnelles même (comme ce fut le cas avec la direction du CNRS) qu'entraîne ce nouveau contexte de programmation, mais aussi d'exercice, de l'activité de recherche au quotidien sont nombreux. Il ne nous appartient pas d'en faire ici un inventaire, mais il nous revient de participer à la réflexion collective qui en résulte et de tenter d'en analyser la signification en regard du projet éditorial de la revue autour de la question interdisciplinaire. D'une certaine façon, NSS a déjà anticipé sur cette réflexion en étant porte-parole des expériences de transgression, aussi bien disciplinaires qu'institutionnelles, que suppose souvent la mise en œuvre de la démarche interdisciplinaire. Le dossier ouvert sur ce sujet en 2004 fera l'objet d'une analyse dans l'un de nos prochains numéros.

Beaucoup de questions soulevées dans les débats actuels ou de craintes exprimées nous interpellent. Des voix s'élèvent - et pas n'importe lesquelles : Académie des sciences, Cour des comptes, ou des personnalités ayant eu de grandes responsabilités dans la direction des organismes de recherche - pour appeler à une refonte radicale du système de recherche, passant par une redistribution des fonctions propres à chaque institution. Or, on peut se demander si ce processus n'est pas d'ores et déjà engagé. La place croissante que prend l'ANR dans le budget affecté aux programmes (supérieur à celui que le CNRS affecte à ses équipes et en augmentation de $70 \%$ cette année) en fait un pilote incontournable, au point que nombreux sont ceux qui y voient une entreprise de réduction du rôle des organismes de recherche, en particulier le CNRS appelé à être cantonné dans le rôle d'une agence de moyens. Une interrogation du même ordre porte également sur l'AERES, appareil de décision et d'orientation des critères d'évaluation des équipes, des chercheurs et des enseignants-chercheurs, qui, au nom d'une standardisation et d'une généralisation des procédures d'évaluation, est susceptible de normaliser des dispositifs adaptés jusqu'ici à la spécificité de chacun des établissements. On sait par ailleurs que les universités, dont la Conférence des présidents (CPU) s'est prononcée pour un statut unique d'enseignant-chercheur, ambitionnent une place centrale dans les activités de recherche, amplifiant en cela la transformation des organismes de recherche en agences de moyens. La crise qu'a connue le plus grand $\mathrm{d}^{\prime}$ entre eux tient pour partie à l'appréciation que l'on peut porter sur les réformes proposées, comme la régionalisation de la direction scientifique pour le développement de pôles plus visibles et plus importants avec les universités : celles-ci allaient-elles dans le sens du renforcement de l'organisme par sa rénovation, comme l'affirmaient ses promoteurs, ou bien contribuaient-elles à son affaiblissement, l'horizon final étant une disparition à terme?

Dans ce tohu-bohu institutionnel, se joue une réponse à la question : "Quelles recherches voulons-nous ? ", qui n'est pas sans rappeler les débats sur le «modèle social français ». Les premières questions que le mouvement SLR a fait surgir - on l'a un peu oublié - ont porté d'emblée sur la conception de l'activité de recherche, en particulier sur la recherche qualifiée de fondamentale et sur les conditions organisationnelles et institutionnelles de son 
épanouissement. La part des corporatismes n'est sans doute pas étrangère à cette revendication d'un modèle spécifique que l'on veut opposer à celui prévalant dans le monde anglo-saxon où prédominent la concurrence et le fonctionnement par projet, autrement dit une programmation et une organisation fondées sur une forme de fragilité en vue d'une plus grande efficacité. L'alignement sur les standards (publications, polarisations, etc.) à l'échelle de la communauté scientifique mondiale, dominée par le monde anglo-saxon, est indéniable, l'ANR ayant vocation à devenir l'équivalent des agences des grands pays comme la National Science Foundation aux États-Unis. Mais le refus d'une telle évolution ou l'ambition de ne pas passer par pertes et profits les acquis d'un système de recherche basé sur des laboratoires assurant, par la stabilité, une continuité au bénéfice du long terme, ne doivent pas faire oublier les grandes inerties et même les injustices qui, entre laboratoires richement dotés ou pas, entre grandes écoles et universités, ne plaident pas pour un statu quo de résistance.

Comment se satisfaire, en effet, de cette incapacité des organismes de recherche à traduire concrètement les ambitions interdisciplinaires affichées? Par exemple, les difficultés passées du CNRS à stabiliser sur le plan structurel, comme ce fut envisagé avec la mise en place de l'INSUE, les programmes interdisciplinaires qu'il avait su impulser, celles plus récentes pour créer un département Environnement et Développement durable qui ne soit pas perçu par les autres départements, en raison de sa vocation pluridisciplinaire et transversale, comme un concurrent, sont là pour nous rappeler les échecs face aux défis auxquels nous sommes confrontés. Car les enjeux sont de taille autour des liens entre Natures, Sciences et Sociétés, comme nous le rappelions dans un éditorial récent (NSS, 14, 1, 2006).

Que penser de ces difficultés et de ces errements, alors que d'autres agences de l'action publique, comme la Banque mondiale ou la Commission européenne, mobilisent sans complexe la communauté scientifique pour s'approprier son expertise? Des objectifs politiques comme la réduction de la pauvreté dans les objectifs du Millénaire des Nations unies ou la Stratégie de Lisbonne s'appuient ainsi sur une science et une expertise au service d'enjeux économiques, validant en outre une démarche que l'on qualifie de «verdissement », puisqu'il faut de plus, et de plus en plus, respecter les dimensions environnementales comme condition du devenir de la planète. L'enjeu n'est plus seulement celui d'une recherche au service d'intérêts privés derrière les classiques injonctions à l'innovation, mais celui d'un pouvoir politique sur la connaissance pour agir dans un monde "postmoderne » hautement technologisé, dans lequel s'entremêlent biens publics et biens privés! À leur façon, ces agences ont compris que connaissance et action ne sont pas séparables...

L'interdisciplinarité qu'appellent de leurs vœux ces agences publiques est-elle du même ordre que celle que tentent d'inventer les pratiques de la recherche scientifique? Car, de ce point de vue, les ambiguïtés ne manquent pas selon que l'on aborde les enjeux, comme les agences, en termes de problem solving - donnant la priorité aux démarches et aux connaissances directement opérationnelles dans un souci de résoudre des problèmes - ou plutôt en termes de problem finding privilégiant la construction des questions ainsi que les démarches et les connaissances qui permettent d'y répondre. $\mathrm{N}^{\prime} \mathrm{y}$ a-t-il pas là explication à un malaise grandissant face à la manière dont la recherche est gouvernée?

NSS est un témoin privilégié des expériences interdisciplinaires des vingt dernières années. Parce que la pratique interdisciplinaire s'est construite dans des programmations organisées dans des logiques de projet fédérant de nombreux partenaires et institutions, parce que les problèmes de l'évaluation de ces pratiques, que ce soit à l'échelle des individus ou des collectifs, ont été constants mais régulièrement débattus, parce que le lien entre la recherche fondamentale et son application a été mis à l'épreuve au travers d'approches en termes de recherche-action ou bien d'une implication des acteurs sociaux dans la construction même de la démarche interdisciplinaire, parce que les questions de formation à l'interdisciplinarité ont été souvent posées, les communautés scientifiques qui ont accompagné le projet éditorial de la revue ont une forte légitimité pour participer aux débats actuels sur l'avenir de notre système de recherche. Ce sera l'objet des prochaines Journées de l'association NSS Dialogues, cette année comme l'année prochaine. C'est notre façon à nous de nous engager dans le processus actuellement en cours de refonte des systèmes de recherche à l'échelle internationale.

Jean-Paul Billaud

Bernard Hubert 